\title{
Stimulatory effects of sorafenib on human non-small cell lung cancer cells in vitro by regulating MAPK/ERK activation
}

\author{
YA-NIAN ZHANG ${ }^{1}$, XIAO-YANG WU ${ }^{1}$, NING ZHONG ${ }^{1}$, JUN DENG $^{1}$, LU ZHANG $^{1}$, \\ WEN CHEN ${ }^{1}, \mathrm{XING} \mathrm{LI}^{1}$ and CHONG-JUN ZHONG ${ }^{2}$ \\ ${ }^{1}$ Department of Thoracic Surgery, Kunshan First People's Hospital Affiliated to Jiangsu University, Kunshan, Jiangsu 215300; \\ ${ }^{2}$ Department of Thoracic Surgery, Second Hospital Affiliated to Nantong University, Nantong, Jiangsu 216001, P.R. China
}

Received June 27, 2013; Accepted November 5, 2013

DOI: $10.3892 / \mathrm{mmr} .2013 .1782$

\begin{abstract}
Sorafenib is an inhibitor of a number of intracellular signaling kinases with antiproliferative, anti-angiogenic and pro-apoptotic effects in tumor cells. Sorafenib has been used in the therapy of advanced renal cell carcinoma. In the present study, using two human non-small cell lung cancer (NSCLC)cell lines, A549 and NCI-H1975, the effects of sorafenib on proliferation, apoptosis and intracellular signaling were systematically characterized. The results revealed that at a low concentration $(5 \mu \mathrm{M})$ and early time point $(6 \mathrm{~h})$, sorafenib is capable of significantly stimulating proliferation of A549 cells, but not NCI-H1975 cells. In addition, the comparison of the two cell lines revealed different cell cycle redistribution and apoptotic susceptibility to sorafenib at this concentration and time point. Western blot analysis revealed that sorafenib upregulated the expression of cyclin D1 and cyclin-dependent kinase 2 and downregulated the expression of BAX at this specific point. Furthermore, sorafenib was confirmed to regulate the expression of cyclin D1 and apoptosis-associated proteins through the regulation of extracellular signal-regulated kinase 1/2 phosphorylation in A549 cells. These findings suggest that, although sorafenib has the potential for use in the treatment of renal cell carcinoma, this compound may also activate NSCLC cells at a specific time point.
\end{abstract}

\section{Introduction}

Non-small cell lung cancer (NSCLC) accounts for $~ 80-85 \%$ of all lung cancer cases, and is the most common cause of worldwide cancer mortality (1). Platinum-based combination

Correspondence to: Professor Chong-Jun Zhong, Department of Thoracic Surgery, Second Hospital Affiliated to Nantong University, Haier Road, Nantong, Jiangsu, 216001, P.R. China

E-mail: sunkekang@126.com

Key words: sorafenib, non-small cell lung cancer cell, mitogen-activated protein kinase, extracellular signal-regulated kinase regimens offer a modest, but significant, survival advantage to NSCLC patients with advanced or metastatic disease, although the majority of patients eventually experience disease progression (2). Lung cancer is a heterogeneous disease with multiple signaling pathways influencing tumor cell survival and proliferation, and it is likely that blocking only one of these pathways allows others to act as salvage or escape mechanisms for cancer cells (3-5). Whether combined inhibition therapy has greater antitumor activity compared with single inhibition therapy is a matter of debate.

Sorafenib is a multi-kinase inhibitor that was originally developed as an inhibitor of RAF-1, a component of the extracellular signal-regulated kinase 1/2 (ERK1/2) pathway, but which was subsequently shown to inhibit multiple other kinases, including class III tyrosine kinase receptors, such as platelet-derived growth factor, vascular endothelial growth factor receptors 1 and 2, c-Kit and FLT3. Sorafenib is currently approved for the treatment of metastatic renal cell carcinoma and for advanced hepatocellular carcinoma $(6,7)$.

Molecularly-targeted therapies, including sorafenib, which disrupts molecular defects in signaling pathways, may provide clinical benefits in the treatment of NSCLC (8). The present study aimed to systematically characterize the in vitro effects of sorafenib on proliferation, apoptosis and intracellular signaling, using two NSCLC cell lines.

\section{Materials and methods}

Cell culture and reagents. The A549 and NCI-H1975 human lung cancer cell lines were purchased from the American Type Culture Collection (Manassas, VA, USA). Cells were maintained in Dulbecco's modified Eagle's medium supplemented with $10 \%$ fetal calf serum, L-glutamine $(5 \mathrm{mmol} / \mathrm{l})$, non-essential amino acids $(5 \mathrm{mmol} / \mathrm{l})$, penicillin $(100 \mathrm{U} / \mathrm{ml})$, and streptomycin $(100 \mathrm{U} / \mathrm{ml})$ (Invitrogen Life Technologies, Carlsbad, CA, USA) at $37^{\circ} \mathrm{C}$ in a humidified $5 \% \mathrm{CO}_{2}$ atmosphere. The mitogen-activated or extracellular signal-regulated protein kinases 1 and 2 (MEK1/2) inhibitor, U0126, was obtained from Cell Signaling Technology (Beverly, MA, USA). Sorafenib was purchased from Bayer Corporation (West Haven, CT, USA) and dissolved in dimethyl sulfoxide (DMSO) with cell medium to the given concentration, with a final DMSO concentration of $0.1 \%$. 
Inhibitor treatment. Stock solutions of sorafenib and U0126 were prepared at appropriate concentrations in DMSO and stored at $-20^{\circ} \mathrm{C}$. For treatment, inhibitor solutions were diluted $1: 1,000$ to appropriate working concentrations (5 or $20 \mu \mathrm{mol} / \mathrm{l}$ cyclopamine and $3 \mu \mathrm{mol} / \mathrm{l}$ U0126) in serum-free medium. Control cultures received medium containing the solvent DMSO at a concentration of $0.1 \%$.

Cell viability assay. Cell proliferation was determined using an MTT viability assay, the most commonly used assay for determining cell growth and death. The MTT survival assay has been described in detail in previous studies (9). Exponentially growing cells were recultured (5,000 cells/well) overnight in 96-well tissue culture plates. Up to $20 \mu 1$ MTT (Sigma-Aldrich, St. Louis, MO, USA) was directly added to the media in each well, with a final concentration of $2 \mathrm{mg} / \mathrm{ml}$. After $4 \mathrm{~h}$ incubation, the medium containing MTT was discarded, and $120 \mu \mathrm{l}$ DMSO was added for $10 \mathrm{~min}$. The absorbance was measured using an enzyme-linked immunosorbent assay reader (Bio-Tek, Inc., Winooskie, VT, USA) at $570 \mathrm{~nm}$, with the absorbance at $630 \mathrm{~nm}$ as the background correction. The cell viability is expressed as the percentage of untreated controls. All experiments were performed at least three times.

Apoptosis assay. Apoptosis analysis by a flow cytometer Annexin V-fluorescein isothiocyanate (FITC)/propidium iodide (PI) kit (BD Biosciences, Sparks, MD, USA) was used to measure the percentage of apoptosis induced by sorafenib. Cells were removed with trypsin and collected into centrifuge tubes together with the culture medium. A total of $5 \mu 1$ Annexin V-FITC solution and $10 \mu \mathrm{l}$ PI $(1 \mu \mathrm{g} / \mathrm{ml})$ were added to these cells for $30 \mathrm{~min}$ in the dark. Flow cytometry and Annexin V-FITC apoptosis analysis were performed as previously described (10). Apoptotic rates were calculated from 10,000 cells using ModFit LT software with FACSCalibur (both Becton Dickinson, San Jose, CA, USA).

Cell cycle assays. The cells were removed with trypsin and collected in centrifuge tubes, together with the culture medium. The contents were centrifuged for $5 \mathrm{~min}$ at $1,800 \mathrm{x}$. The supernatant was poured out, washed once with $1 \mathrm{X}$ phosphate-buffered saline (PBS), and centrifuged for a subsequent $5 \mathrm{~min}$. The cells were then fixed with $5 \mathrm{ml}$ pre-cooled $70 \%$ ethanol for at least $4 \mathrm{~h}$. The fixed cells were centrifuged and washed with $1 \mathrm{X}$ PBS. Following centrifugation, the cell pellets were resuspended in $500 \mu \mathrm{l} \mathrm{PI}(10 \mu \mathrm{g} / \mathrm{ml})$ containing $300 \mu \mathrm{g} / \mathrm{ml}$ RNase (Sigma-Aldrich). The cells were incubated on ice for $30 \mathrm{~min}$, and filtered with a $53 \mu \mathrm{m}$ nylon mesh. The cell cycle distribution was calculated from 10,000 cells using ModFit LT software (Becton Dickinson) using FACSCalibur.

Western blot assay. Cell lysates were prepared and western blot analysis was performed as previously described (10). Equal aliquots of total cell protein (50 $\mu \mathrm{g} /$ lane) were electrophoresed on sodium dodecyl sulfate-polyacrylamide gels, transferred onto polyvinylidene fluoride membranes, and blotted using the primary antibodies: $\beta$-actin (C-4), cyclin D1 (A-12), cyclin-dependent kinase (CDK)2 (M2), nuclear factor (NF)- $\mathrm{B}$ (P65A), matrix metallopeptidase (MMP)-2 (2C1), MMP-9
(6-6B), BAX (P-19) and Bcl-2 (C-2; all 1:1,000 dilution; Santa Cruz Biotechnology, Inc., Santa Cruz, CA, USA), followed by the secondary antibodies horseradish peroxidase-labeled goat anti-mouse (GAM-007) and goat anti-rabbit (SC-2004) IgG, and phospho-ERK1/2 (T202/Y204) and ERK1/2 (1:1,000 dilution; Cell Signaling Technology, Beverly, MA, USA). The protein bands were visualized using an enhanced chemiluminescence system (Union Bioscience Corporation, Hangzhou, China) with prestained markers as molecular size standards. At least three independent experiments were performed for each cell type studied.

Statistical analysis. Data are presented as the mean \pm standard deviation. Experimental results of the treated and control groups were compared using two-tailed Student's t-test. All statistical tests were performed using SPSS version 17.0 (SPSS Inc., Chicago, IL, USA). $\mathrm{P} \leq 0.05$ was considered to indicate a statistically significant difference.

\section{Results}

Sorafenib decreases breast cancer cell proliferation. MTT viability assays were conducted to elucidate the potential biological effects of sorafenib in different lung cancer cells. As shown in Fig. 1A, the NCI-H1975 cells treated with cyclopamine exhibited significant reduction in proliferation rates compared with the control cells at the two concentrations (5 and $20 \mu \mathrm{M}$ ), and sorafenib also markedly inhibited A549 cells at a concentration of $20 \mu \mathrm{M}$. However, at a concentration of $5 \mu \mathrm{M}$ and $6 \mathrm{~h}$ time point, the proliferation rate was increased compared with the control cells (Fig. 1B).

Effect of sorafenib on cell cycle distribution and apoptosis in different cells. To investigate the different proliferation results in sorafenib-treated lung cancer cells, cell cycle proliferation and apoptosis was examined by flow cytometry. The results showed that sorafenib decreased the proportion of A549 cells in the G1 phase of the cell cycle and increased that of cells in the $\mathrm{S}$ phase (Fig. 2A), and the cell cycle redistribution was not observed in NCI-H1975 cells at this time point (Fig. 2B). The apoptosis assay revealed that sorafenib decreased the proportion of apoptotic cells in A549 cells at the $6 \mathrm{~h}$ time point, which was not observed in NCI-H1975 cells (Fig. 2C and D). These data indicate that cell cycle distribution and apoptosis were involved in the proliferation of sorafenib-treated lung cancer cells.

Sorafenib affects the expression of cell cycle, invasion and apoptosis-associated proteins. The alterations in the cell cycle, invasion- and apoptosis-associated proteins in sorafenib-treated cells were evaluated and compared with the control cells using western blot analysis. As shown in Fig. 3A, the expression levels of cyclin D1, CDK2, NF- $\kappa$ B, MMP2, MMP9 and Bcl 2 decreased significantly in sorafenib-treated NCI H1975 cells. In A549 cells, sorafenib significantly enhanced the expression of cyclinD1 and CDK2, and suppressed BAX expression at the $6 \mathrm{~h}$ time point (Fig. 3B). With regard to invasion-associated proteins, there was no difference between the two cell lines (Fig. 3C and D). Sorafenib regulates the proliferation and apoptosis of A549 cells by activating 

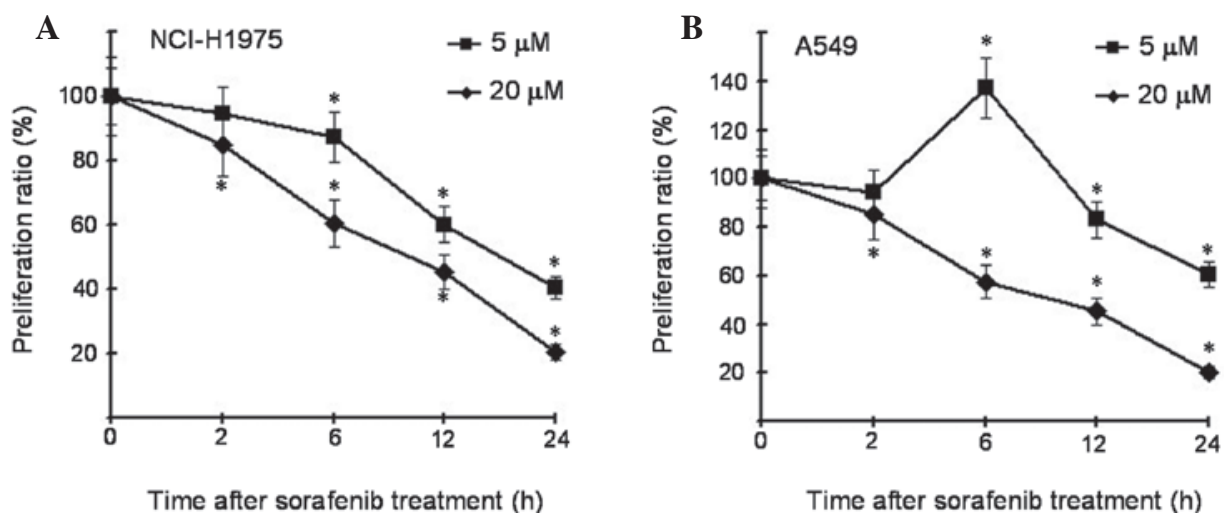

Figure 1. Effects of sorafenib (5 and $20 \mu \mathrm{M})$ on the proliferation of different human lung cancer cell lines (A549 and NCI-H1975). (A and B) The indicated cell lines were cultured for $24 \mathrm{~h}$ in complete culture medium containing specific concentrations of sorafenib, following which cell viability was assessed with the MTT assay. All samples were prepared in triplicate. The proliferation rate was measured as fold changes in cell growth. ${ }^{*} \mathrm{P}<0.05$, vs. the control group.

A

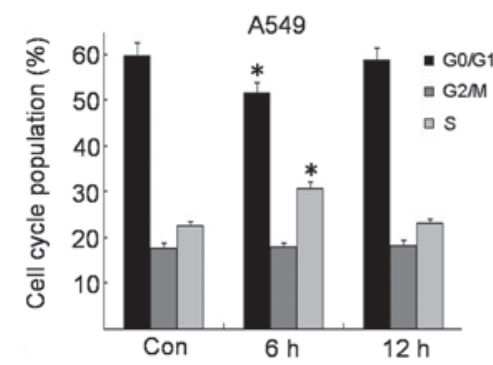

C

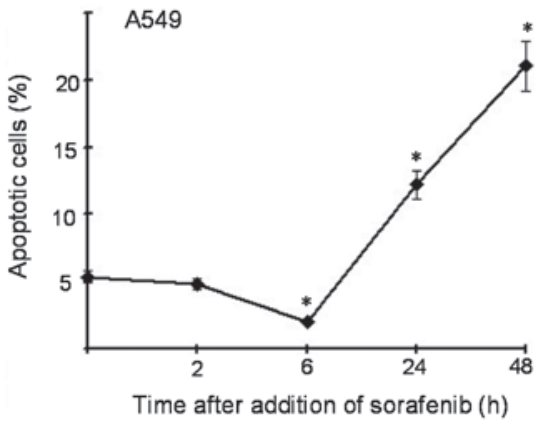

B
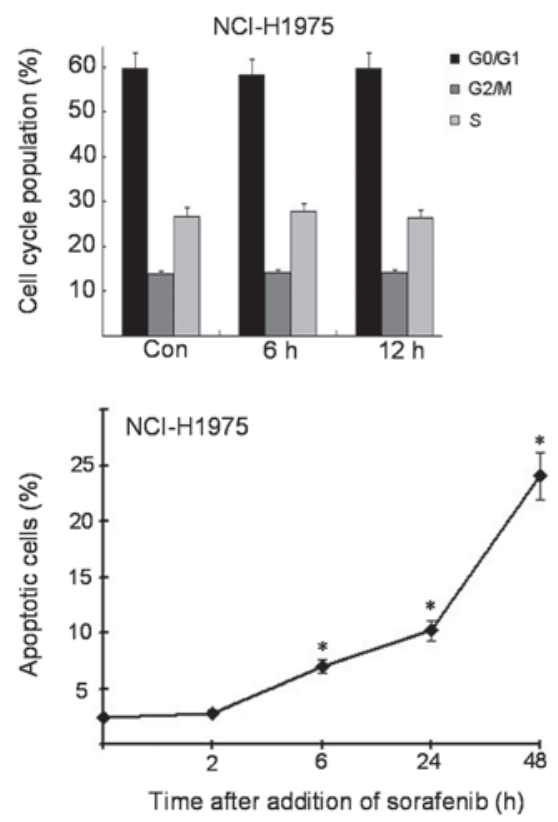

Figure 2. Effects of sorafenib on cell cycle distribution and apoptosis in NSCLC cells. (A and B) Cells were incubated for 0, 6, or 12 h in complete culture medium containing $5 \mu \mathrm{M}$ sorafenib and were fixed, stained with PI, and analyzed for cell cycle distribution by flow cytometry. (C and D) Cells were incubated for $0,2,6,24$ or $48 \mathrm{~h}$ in complete culture medium containing $5 \mu \mathrm{M}$ sorafenib and were fixed, stained with Annexin V-FITC/PI and analyzed for apoptosis by flow cytometry. All samples were prepared in triplicate. ${ }^{*} \mathrm{P}<0.05$, vs. the control group. NSCLC, non-small-cell lung cancer; PI, propidium iodide; FITC, fluorescein isothiocyanate.

the ERK1/2 signaling pathway. To examine the effects of sorafenib on the ERK1/2 signaling pathway in A549 cells, immunoblot analysis was performed with antibodies specific for phosphorylated ERK1/2. The results of this study reveal that sorafenib markedly enhanced ERK phosphorylation in A549 cells at the $5 \mu \mathrm{M}$ concentration and $6 \mathrm{~h}$ time point, but had no marked effect on the expression of this protein (Fig. 4). Phosphorylation of ERK1/2 is a key initial response to cell cycle distribution and apoptosis. With regard to downstream proteins, sorafenib markedly upregulated the expression of cyclin D1, and inhibited the expression of BAX at the same concentration and time point. The specific signaling cascade involved in this response was investigated using the MEK1/2 inhibitor (U0126) specific to the MAPK/ERK pathway. The increased phospho-ERK1/2 following sorafenib-treatment was inhibited by U0126, as well as the downstream protein cyclin D1, Bcl-2 and BAX (Fig. 4), which suggests that sorafenib mediates cell cycle distribution and apoptosis by modulating the ERK signaling pathway.

\section{Discussion}

NSCLC remains a leading cause of mortality worldwide among patients diagnosed with malignancies (11). The standard therapeutic concept of lung cancer is based on cisplatin chemotherapy. However, during the last few decades, no significant improvement in the prognosis of patients with lung cancer has been achieved (12).

Sorafenib, an oral multi-kinase inhibitor, inhibiting serine/threonine Raf kinases and RTKs, including vascular 
A

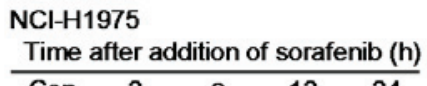

\begin{tabular}{lllll}
\hline Con & 2 & 6 & 12 & 24
\end{tabular}
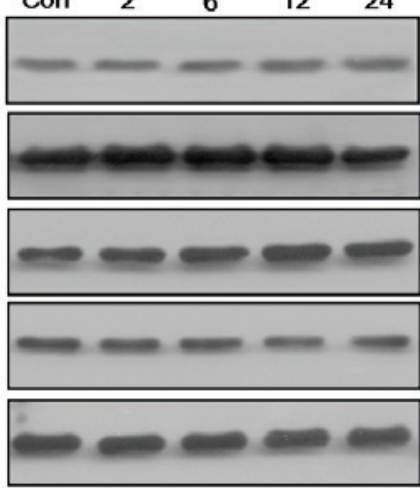

C

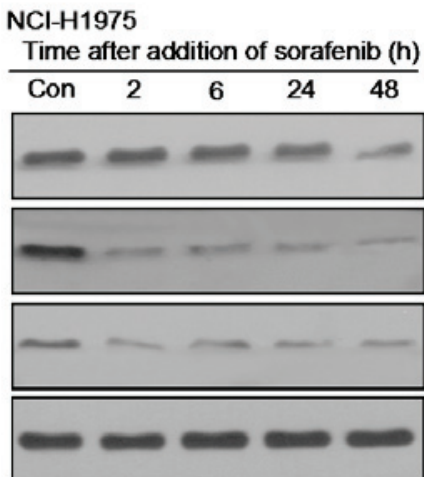

B

A549

Time after addition of sorafenib (h)

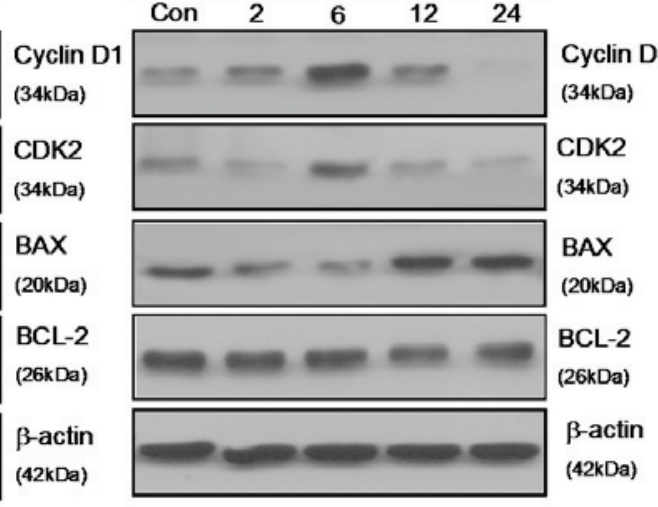

D $A 549$

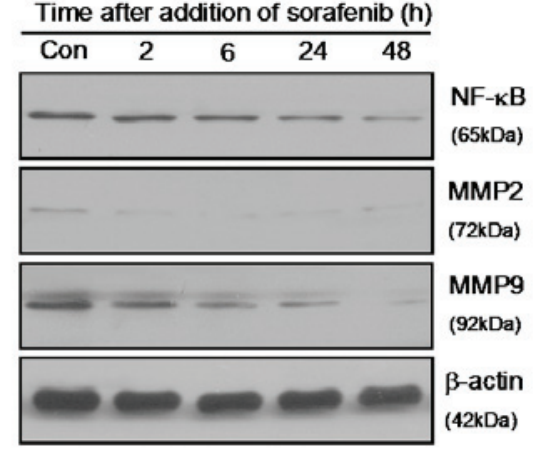

NF-kB
(65kDa)
MMP2
(72kDa)
MMP9
(92kDa)
(3-actin
(42kDa)

Figure 3. Effects of sorafenib on the expression of cell cycle-, invasion- and apoptosis-associated proteins. (A and B) Whole cell lysates of sorafenib-treated A549 and NCI-H1975 cells were prepared, and $50 \mu \mathrm{g}$ proteins were resolved using SDS-PAGE, followed by immunoblotting with the indicated specific antibodies against NF- $\mathrm{KB}$, MMP2, MMP9 cyclin D1 and CDK2. (C and D) Specific antibodies against BAX and Bcl-2 were used to determine the effect of sorafenib on apoptosis-associated proteins. NF-кB, nuclear factor- $\mathrm{B}$; MMP9, matrix metallopeptidase 9; CDK2, cyclin-dependent kinase 2

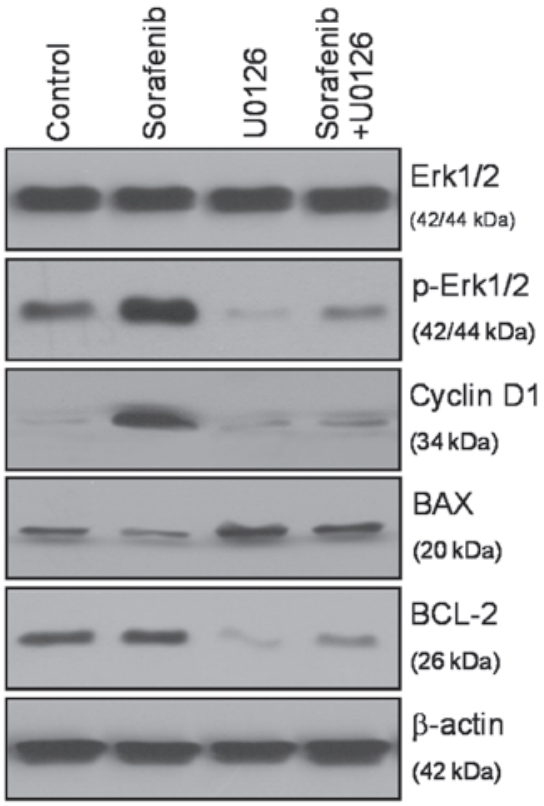

Figure 4. Sorafenib modulated the proliferation and apoptosis in A549 cells via MAPK/ERK signal pathway. Western blot assays were performed to determine phosphorylated ERK1/2 and the downstream cascades in sorafenib-treated A549 cells. At the same time point A549 cells treated with sorafenib were cultured in the presence of the MEK 1/2 inhibitor U0126. The expression level of phospho-ERK1/2 and downstream cyclin D1 and BAX were determined by western blot analysis. Blots are representative of three experiments. MAPK, mitogen-activated protein kinase; ERK1/2, extracellular signal-regulated kinase $1 / 2$. endothelial growth factor receptor and platelet-derived growth factor receptor-b, is currently used for the therapy of advanced renal carcinoma. Furthermore, antitumor activity and an improved outcome have also been observed for patients with other carcinomas, including hepatocellular carcinoma and NSCLC (6,7,13-16).

In the present study, it was observed that at a low concentration and early time point, sorafenib is capable of significantly stimulating the proliferation of A549 cells, which was not observed in NCI-H1975 cells. The cell cycle assay showed that sorafenib decreased the proportion of A549 cells in the G1 phase of the cell cycle, and increased that of cells in the S phase, which was different with NCI-H1975 cells. The apoptosis assay also revealed that sorafenib decreased the proportion of apoptotic cells in A549 cells at a specific time point.

Phosphorylated ERK is a key downstream target of the Ras/Raf/MEK/ERK signaling pathway, and dysregulation of this pathway occurs in approximately one-third of all human cancers (17). ERK is the terminal kinase in this pathway. During the phosphorylation cascade, ERK1/2 is activated by mitogen-activated protein kinases. Mitogen-stimulated cell growth, through survival/apoptosis and cell cycle control, is frequently linked to phosphorylated ERK translocation from the cytoplasm to the nucleus and to the initiation of transcriptional activation of cell regulatory genes $(18,19)$. Therefore, the current study focused on the effects of sorafenib on the phosphorylation status of ERK1/2. As described in the results, 
high concentrations of sorafenib decreased the phosphorylation levels of ERK-1/2. Notably, a significant stimulatory effect of sorafenib was observed at low concentrations on ERK-1/2 phosphorylation. To further elucidate the underlying signaling pathways, the MEK inhibitor U0126 was used. The results showed that the increased phospho-ERK1/2 following sorafenib-treatment was inhibited by U0126, as well as the downstream protein cyclin D1, Bcl-2 and BAX, which suggests that sorafenib mediates cell cycle distribution and apoptosis by modulating the ERK signaling pathway.

The current observations demonstrate the antimigratory and antiproliferatory effects of sorafenib in different human NSCLC cells. However, the stimulatory effects at low concentrations of sorafenib in certain cancer cells may have marked consequences for this antitumor drug. This may be particularly relevant for selecting dosing regimens that avoid low plasma concentrations of sorafenib in order to optimize the treatment with this promising antitumor drug.

\section{References}

1. Kyle F and Spicer J: Targeted therapies in non-small cell lung cancer. Cancer Imaging 8: 199-205, 2008.

2. Ray MR, Jablons D and He B: Lung cancer therapeutics that target signaling pathways: an update. Expert Rev Respir Med 4: 631-645, 2010

3. Zhang X, Li Y, Li H, et al: Combined EGFR and VEGFR versus single EGFR signaling pathways inhibition therapy for NSCLC: a systematic review and meta-analysis. PLoS One 7: e40178, 2012.

4. Larsen JE, Cascone T, Gerber DE, et al: Targeted therapies for lung cancer: clinical experience and novel agents. Cancer J 17: 512-527, 2011.

5. Pal SK, Figlin RA and Reckamp K: Targeted therapies for non-small cell lung cancer: an evolving landscape. Mol Cancer Ther 9: 1931-1944, 2010.

6. Ramakrishnan V, Timm M, Haug JL, et al: Sorafenib, a multikinase inhibitor, is effective in vitro against non-Hodgkin lymphoma and synergizes with the mTOR inhibitor rapamycin. Am J Hematol 87: 277-283, 2012.
7. Wilhelm S, Carter C, Lynch M, et al: Discovery and development of sorafenib: a multikinase inhibitor for treating cancer. Nat Rev Drug Discov 5: 835-844, 2006.

8. Wilhelm SM, Adnane L, Newell P, et al: Preclinical overview of sorafenib, a multikinase inhibitor that targets both Raf and VEGF and PDGF receptor tyrosine kinase signaling. Mol Cancer Ther 7: 3129-3140, 2008.

9. Jiao Y, Sun KK, Zhao L, et al: Suppression of human lung cancer cell proliferation and metastasis in vitro by the transducer of ErbB-2.1 (TOB1). Acta Pharmacol Sin 33: 250-260, 2012

10. Sun KK, Zhong N, Yang Y, Zhao L and Jiao Y: Enhanced radiosensitivity of NSCLC cells by transducer of erbB2.1 (TOB1) through modulation of the MAPK/ERK pathway. Oncol Rep 29: 2385-2391, 2013.

11. Scagliotti G, Novello S, von Pawel J, et al: Phase III study of carboplatin and paclitaxel alone or with sorafenib in advanced non-small-cell lung cancer. J Clin Oncol 28: 1835-1842, 2010.

12. Bearz A, Berretta M, Lleshi A and Tirelli U: Target therapies in lung cancer. J Biomed Biotechnol 2011: 921231, 2011.

13. Rose A, Grandoch M, vom Dorp F, et al: Stimulatory effects of the multi-kinase inhibitor sorafenib on human bladder cancer cells. Br J Pharmacol 160: 1690-1698, 2010.

14. Bareford MD, Park MA, Yacoub A, et al: Sorafenib enhances pemetrexed cytotoxicity through an autophagy-dependent mechanism in cancer cells. Cancer Res 71: 4955-4967, 2011.

15. Takahashi O, Komaki R, Smith PD, et al: Combined MEK and VEGFR inhibition in orthotopic human lung cancer models results in enhanced inhibition of tumor angiogenesis, growth, and metastasis. Clin Cancer Res 18: 1641-1654, 2012.

16. Takezawa K, Okamoto I, Yonesaka K, et al: Sorafenib inhibits non-small cell lung cancer cell growth by targeting B-RAF in KRAS wild-type cells and C-RAF in KRAS mutant cells. Cancer Res 69: 6515-6521, 2009.

17. Marampon F, Gravina GL, Di Rocco A, et al: MEK/ERK inhibitor U0126 increases the radiosensitivity of rhabdomyosarcoma cells in vitro and in vivo by downregulating growth and DNA repair signals. Mol Cancer Ther 10: 159-168, 2011.

18. Klein PJ, Schmidt CM, Wiesenauer CA, et al: The effects of a novel MEK inhibitor PD184161 on MEK-ERK signaling and growth in human liver cancer. Neoplasia 8: 1-8, 2006.

19. Shukla A, Hillegass JM, MacPherson MB, et al: ERK2 is essential for the growth of human epithelioid malignant mesotheliomas. Int J Cancer 129: 1075-1086, 2011. 